\title{
Fingerprints for Machines - Characterization and Optical Identification of Grinding Imprints
}

\author{
Ralf Dragon $^{1}$, Tobias Mörke ${ }^{2}$, Bodo Rosenhahn ${ }^{1}$, and Jörn Ostermann ${ }^{1}$ \\ 1 Institut für Informationsverarbeitung \\ \{dragon, rosenhahn, ostermann\}@tnt.uni-hannover.de \\ ${ }^{2}$ Institute of Production Engineering and Machine Tools \\ moerke@ifw.uni-hannover.de \\ Leibniz Universität Hannover, Germany
}

\begin{abstract}
The profile of a $10 \mathrm{~mm}$ wide and $3 \mu \mathrm{m}$ deep grinding imprint is as unique as a human fingerprint. To utilize this for fingerprinting mechanical components, a robust and strong characterization has to be used. We propose a feature-based approach, in which features of a 1D profile are detected and described in its 2D space-frequency representation. We show that the approach is robust on depth maps as well as intensity images of grinding imprints. To estimate the probability of misclassification, we derive a model and learn its parameters. With this model we demonstrate that our characterization has a false positive rate of approximately $10^{-20}$ which is as strong as a human fingerprint.
\end{abstract}

\section{Introduction}

For more than one century, many mechanical components are manufactured interchangeable. This allowed mass production with enhanced quality at lower costs. However interchangeable parts are usually indistinguishable and thus not identifiable. This leads to problems with product plagiarism and the determination of origin of a component. Labeling components is often not a suitable approach as labels may get lost, copied or change functional properties of a component. Thus, the variation of inherent material properties is used for fingerprinting in various fields, e.g. chemical fingerprints [10] for marking medicine

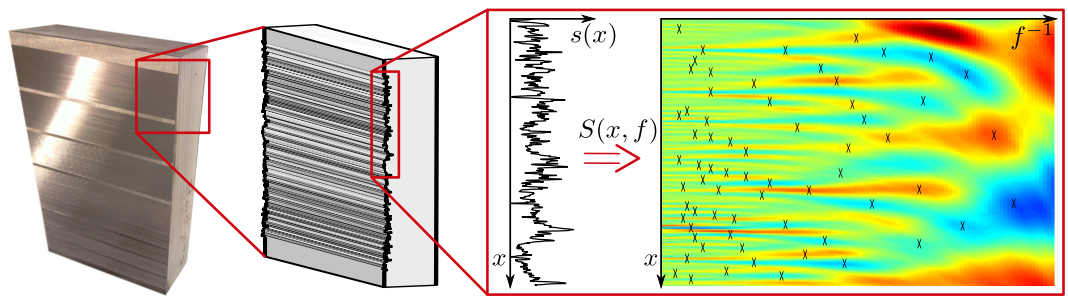

Fig. 1: Approach: Profile $s(x)$ of a grinding imprint image is obtained and characteristic features are extracted in the space-frequency domain $S(x, f)$. 
or Physical Unclonable Functions [14] for the identification of computer chips. In this work, grinding imprints are characterized for fingerprinting mechanical components (cf. Fig. 1). They are created by a grinding wheel which is shifted in grinding direction. The imprints here have a width of $10 \mathrm{~mm}$ and a depth standard deviation of $\sigma \approx 3 \mu \mathrm{m}$. To enforce statistically independent fingerprints, the grinding wheel is straightened before an imprint is created, which means that several layers of abrasive materials are removed from its surface.

In this paper, we derive the framework to extract, to characterize and to verify such imprints in order to identify components. There are four requirements for the characterization. It should be (a) independent to geometric variations of the imprint such as a shift or a scale, as a a highly-precise alignment of extracted data should not be necessary, (b) robust to a few but maybe strong local perturbations, and (c) unique like a human fingerprint. As we want to allow that the characterization is computed from depth maps as well as from intensity images, the characterization should also be (d) independent from a non-linear scale in depth. In our approach, the characteristic feature constellation is learned in the space-frequency domain of the imprint profile. Our contributions are

- the detection of features of a $1 \mathrm{D}$ grinding profile in the $2 \mathrm{D}$ continuous wavelet space

- the characterization and matching of profiles using feature locations and descriptions, and

- the analysis of the strength of our fingerprint characterization with respect to false positive detection and surface perturbation.

By this, we demonstrate that our approach allows identification as secure as if a human fingerprint was analyzed.

\subsection{Related Works}

Historically, fingerprints are characterized by the constellation of minutiae which are ridge properties like crossings, bifurcations, dots and endings. The strength of a fingerprint is due to the fact that the constellation of the minutiae is unique. The probability of a false positive classification with only 12 correspondences is that low $\left(\approx 10^{-20}\right)$ that it is sufficient as evidence in court [11].

In our approach we follow the idea of detecting minutiae. As the analyzed imprint does not contain explicit patterns like ridge crossings, we use an approach inspired by feature-based image analysis, where generalized salient features are detected. These features may be maxima in the DoG scale space [6], anisotropic blobs [9], homogeneous regions [8] or maxima of local entropy [5]. Then, analog to the minutiae type, a descriptor is built which usually is an affine and illumination invariant representation of the local image contents. To verify the constellation of matching descriptors, usually an affine or projective transformation is fitted to the correspondences using RANSAC [2]. However, grinding imprints are not suitable for feature-based approaches as they vary slowly in the grinding direction and abruptly orthogonal to it. This means that an imprint is better characterized using its 1D profile. Thus, we focus on features of grinding imprint profiles. 
Traditionally, grinding imprints are characterized according to ISO 25178, which describes the rules and procedures for the assessment of surface texture. However, such global surface texture parameters such as the root mean square height of the surface $S_{q}$ or the maximum height of the surface $S_{z}$ can neither be used to distinguish nor to describe surfaces robustly.

Further, methods of statistical texture analysis could be used to characterize an imprint. E.g. in [13], the maxima locations of an entropy measure over different orders and scales is used as characteristic fingerprint image. But as the relevant information is contained in one dimension, statistical methods for signal analysis in one dimension seem more adequate.

The differences between audio data and grinding profiles are the statistical signal properties - grinding profile samples are highly transient- and the number of samples -a profile consist only of 3200 samples whereas audio data usually of $10 \mathrm{~s}$ or more at $44 \mathrm{kHz}$. Most approaches tackle audio fingerprinting as a pattern recognition problem where feature vectors are to be classified. These are usually regularly sampled from the distribution of spectral coefficients like Fourier Coefficients, Mel Frequency Cepstral Coefficients or Wavelet Coefficients. For a broad overview we refer to [1]. In contrast to this, we decided to detect specific salient positions in the space-frequency domain and describe them locally. This has the advantage that signals with change in scale or with partial differences are better comparable.

A similar idea has recently been introduced for audio indexing [15]. They analyze the Short Time Fourier Transform (STFT) spectrogram and use customary SIFT [6] features for its description. However as imprint profiles are more transient than audio data, the STFT is not a stable characterization. Further SIFT is not a suitable approach for describing features as rotation invariance is not desirable. Nonetheless, in Experiment 4.4, we use this method for a comparison with our proposed approach.

There exist many applications in which the continuous wavelet transformation [7] (CWT) is used to compare 1D data. In [3], CWTs of two time series are multiplied to analyze the correlation between different climatic effects. The same method is applied in [12] to analyze economic relationships. However to the best of our knowledge, besides [15], there is no approach in which salient features in the space-frequency domain are detected and compared.

The outline of this paper is as follows: In Section 2, our feature-based profile characterization approach is explained. We derive an estimate on the probability of falsely matching two profiles in Section 3. We evaluate the properties of the approach in experiments in Section 4. A conclusion is given in Section 5.

\section{Feature-based Profile Characterization}

\subsection{Detection}

First, the profile $s(x)$ is extracted from the 2D depth map or intensity image $d(x, y)$ of the imprint (cf. Fig. 1). We assume that $x$ is the dimension orthogonal 
to the grinding. To cancel out noise and outliers, $s(x)$ is built by averaging over $n_{y}$ image rows around $y=y_{0}$ :

$$
s(x)=\frac{1}{n_{y}} \sum_{y=y_{0}-n_{y} / 2}^{y_{0}+n_{y} / 2} d(x, y) .
$$

$s(x)$ is transformed to the space-frequency domain $S(x, f)$ using the continuous wavelet transform [7] (CWT) with the wavelet $\phi_{x, f}(\xi)$ :

$$
S(x, f)=\int_{-\infty}^{\infty} s(\xi) \cdot \phi_{x, f}(\xi) d \xi .
$$

$\phi$ is parametrized by $x$ and $f$, where $x$ is the translation the wavelet is centered around and $f$ is the dominant frequency in the power density spectrum of $\phi_{x, f}$. To cover a wide range of frequencies, the frequency is sampled logarithmically between $\lambda_{0}=s_{c} / 2$ and $\lambda_{1}=s_{x} / 2$, where $s_{x}$ is the profile length and $s_{c}$ the expected diameter of the imprint of one grain, which is $125 \mu \mathrm{m}$ here.

Like the filter response of every linear system, for a constant $f, S(x, f)$ denotes a local estimate of the cross correlation (cf. (2)) of the profile at position $x$ and the wavelet. Peaks in $S$ at $\left(x_{m}, f_{m}\right)$ indicate a high correlation between the local neighborhood of $s\left(x_{m}\right)$ and $\phi_{x_{m}, f_{m}}$. The distribution of peaks (cf. Fig. 1) is specific for each profile and fulfills requirement (b) as local changes do not change the global distribution (cf. Fig. 2).

Wavelets are useful here to obtain sharp-edged and stable peaks in $S(x, f)$. In principle, an arbitrary real-valued wavelet could be used. On the one hand, sharp-peaked wavelet distributions are better locatable in $x$ and $f$. Thus, a wavelet should be shaped like a profile. On the other hand, peaks should occur at different frequencies $f$, so the wavelet should be generic enough. We empirically chose the Daubechies wavelet [7] of order 4 as it matches both criteria, but we did not do extensive evaluation of different wavelets.

As feature location, local maxima are chosen as they are locatable most precisely and robustly under perturbation. The candidate $m$ with coordinates $\left(x_{m}, f_{m}\right)$ is found using non-maximum suppression on $|S(x, f)|$. Then region $R_{m}$ is determined which describes the extents of feature $m$. In order to be scale invariant, its extents are proportional to the wavelet wavelength $\lambda_{m}=f_{m}^{-1}$. In spatial direction, the extent is $x=x_{m} \pm \lambda_{m} / 2$. Similarly, in frequency direction it is chosen such that $f^{-1}=\lambda_{m} \pm \lambda_{m} / 2$. Thus, the borders of $R_{m}$ are

$$
x=\left[x_{m}-\lambda_{m} / 2 ; x_{m}+\lambda_{m} / 2\right], \quad f=\left[2 / 3 f_{m} ; 2 f_{m}\right] .
$$

\subsection{Description}

To avoid clusters in the profile characterization, all candidates $j$ are discarded, if their detected location $\left(x_{j}, f_{j}\right)$ falls into the region of another candidate $i$ with higher absolute value in $S$ :

$$
\left|S\left(x_{j}, f_{j}\right)\right|<\left|S\left(x_{i}, f_{i}\right)\right| \quad \vee \quad\left(x_{j}, f_{j}\right) \in R_{i} .
$$




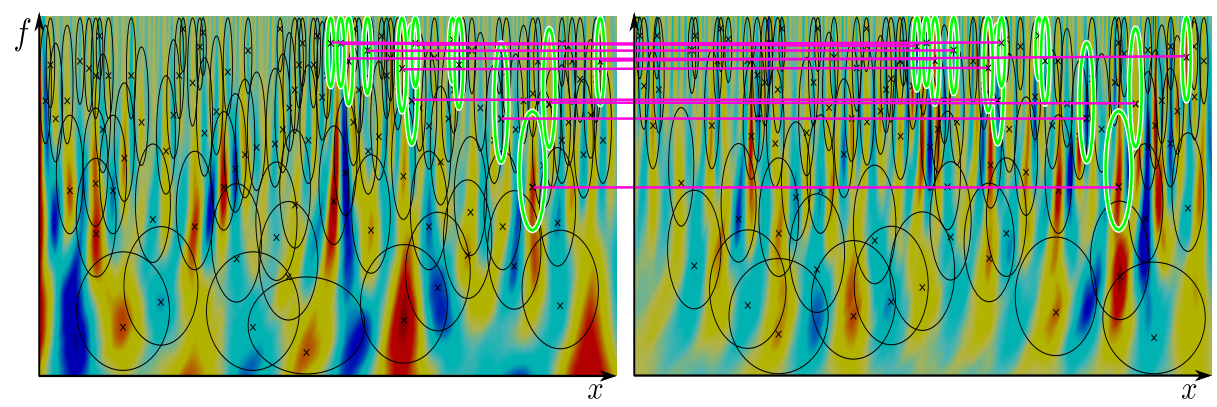

Fig. 2: Detected (black ellipses) and matching (green ellipses) features in the continuous wavelet space $S(x, f)$ between two profiles with partially similar local (right half) but different global structure (lower half).

For all remaining candidates $m$, a descriptor $\boldsymbol{D}_{m}$ is built by sampling $R_{m}$ in equal intervals. In this work, we sample 3 frequencies with 9 samples each resulting in a descriptor length of 27. Next, the descriptor is normalized, such that $\mathrm{E}\left[\boldsymbol{D}_{m}\right]=0$ and $\left\|\boldsymbol{D}_{m}\right\|=1$. As a scaling or a depth shift of the profile has no influence on the descriptor, requirement (d) is achieved. Finally, we have a list of feature locations $\left(x_{m}, f_{m}=1 / \lambda_{m}\right)$ with one descriptor $\boldsymbol{D}_{m}$ for each feature.

\subsection{Comparison}

In this Section, we explain how the characterizations of two profiles $s^{(1)}$ and $s^{(2)}$ are compared. First, all correspondence candidates $(i, j)$ are found whose descriptors $\boldsymbol{D}_{i}^{(1)}$ and $\boldsymbol{D}_{j}^{(2)}$ are similar under the cosine metric:

$$
\cos \left(\angle\left(\boldsymbol{D}_{i}^{(1)}, \boldsymbol{D}_{j}^{(2)}\right)\right)=\frac{\left\langle\boldsymbol{D}_{i}^{(1)}, \boldsymbol{D}_{j}^{(2)}\right\rangle}{\left\|\boldsymbol{D}_{i}^{(1)}\right\| \cdot\left\|\boldsymbol{D}_{j}^{(2)}\right\|}=\left\langle\boldsymbol{D}_{i}^{(1)}, \boldsymbol{D}_{j}^{(2)}\right\rangle>d_{t},
$$

where $d_{t}$ is a threshold set to 0.9 here.

Next, the spatial constellation of all candidates is verified. It is assumed that profile $s^{(1)}$ and $s^{(2)}$ spatially differ by a shift $\Delta_{x}$ and a wavelength ratio $\Delta_{\lambda}$. Thus, the constellation of all correspondence pairs $(i, j)$ must fulfill

$$
\boldsymbol{l}_{j}^{(2)}=\left[\begin{array}{c}
x_{j}^{(2)} \\
\lambda_{j}^{(2)}
\end{array}\right]=\Delta_{\lambda} \cdot\left[\begin{array}{c}
x_{i}^{(1)}+\Delta_{x} \\
\lambda_{i}^{(1)}
\end{array}\right]=\Delta_{\lambda} \cdot\left(\boldsymbol{l}_{i}^{(1)}+\boldsymbol{\Delta}_{x}\right) .
$$

The two unknowns can be estimated from one correspondence pair. They are optimized by minimizing the squared symmetric Euclidean distance

$$
e_{i, j}^{2}=\frac{1}{2}\left|\boldsymbol{l}_{j}^{(2)}-\Delta_{\lambda} \cdot\left(\boldsymbol{l}_{i}^{(1)}+\boldsymbol{\Delta}_{x}\right)\right|^{2}+\frac{1}{2}\left|\boldsymbol{l}_{i}^{(1)}-\boldsymbol{l}_{j}^{(2)} / \Delta_{\lambda}-\boldsymbol{\Delta}_{x}\right|^{2}
$$

over all candidates $(i, j)$ using RANSAC [2]. Outliers are detected if $e_{i, j}$ is bigger than an error radius $r$, which is set to 6 samples here. After this, correspondences with the same geometric constellation have been found. 


\section{$3 \quad$ False Positive Profile Matches}

In this section, we derive a model on the probability $p_{\mathrm{fp}}$, prof of wrongly matching two profiles of different components. We assume that the classification is based on the numbers of correspondences $k$.

For each possible correspondence, there are two criteria in order to be regarded as inlier: According to Section 2.3, first the descriptors have to match. We call the probability of one false descriptor match $p_{\mathrm{fp}}$, desc . Second, a correspondence has to fulfill spatial constraints with a probability of $p_{\text {cstl }}$ in order to be considered as inlier to RANSAC. Assuming statistical independence, the probability of fulfilling both constraints for a random correspondence inlier is

$$
p_{\mathrm{ri}}=p_{\mathrm{fp}, \mathrm{desc}} \cdot p_{\mathrm{cstl}} \cdot
$$

If there are $n_{1}$ features detected in the first profile and $n_{2}$ in the second, there may be $n=n_{1} n_{2}$ correspondences. Assuming $n \gg k$, the probability of exactly $k$ inliers meeting the inlier criteria out of $n$ correspondences can be expressed using the binomial distribution

$$
B\left(k, n, p_{\mathrm{ri}}\right)=\left(\begin{array}{l}
n \\
k
\end{array}\right) \cdot p_{\mathrm{ri}}^{k} \cdot\left(1-p_{\mathrm{ri}}\right)^{n-k} .
$$

However, one of the $k$ correspondences always fulfills the RANSAC motion model as this model is derived from it. This correspondence matches with $p_{\mathrm{fp}}$, desc and there may be $n_{2}$ possible motions derived from it. So the probability of obtaining exactly $k$ correspondences is

$$
p_{\mathrm{k}, \operatorname{prof}}(k)=1-\left(1-p_{\mathrm{fp}, \operatorname{desc}} \cdot B\left(k-1, n-1, p_{\mathrm{ri}}\right)\right)^{n_{2}}, k \geq 2 .
$$

Thus, the distribution of two independent profiles to match using a threshold of $k$ filtered correspondences is

$$
p_{\mathrm{fp}, \operatorname{prof}}(k)=\sum_{i=k}^{n} p_{\mathrm{k}, \operatorname{prof}}(i), k \geq 2 .
$$

\section{Experiments}

\subsection{Evaluation of false positive detections}

We use the model from Section 3 in order to determine the probability of two independent fingerprints to match. The profiles are extracted from depth images taken with a confocal white light microscope which uses depth from focus [4]. Its lateral sampling distance is $3.1 \mu \mathrm{m}$, so there are 3200 samples for the $10 \mathrm{~mm}$ wide profile. The a priori probabilities $p_{\mathrm{fp}, \text { desc }}$ and $p_{\mathrm{cstl}}$ from Eq. (8) are estimated from depth images of 45 different grinding imprints. Of each depth image $d^{(i)}$, two profiles $s_{1}^{(i)}$ and $s_{2}^{(i)}$ are taken of each at a distance of $1 \mathrm{~mm}$. All descriptors 


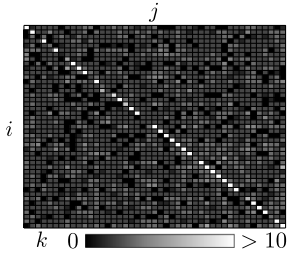

(a)

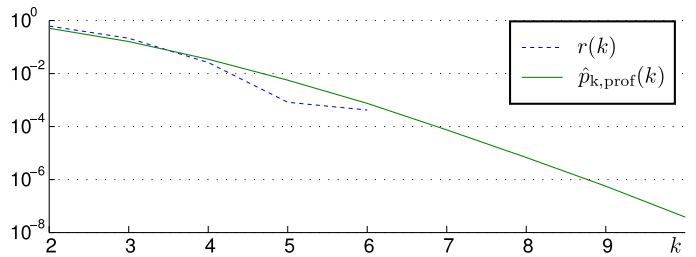

(b)

Fig. 3: (a) Confusion matrix $C$ with number of matches $C(i, j)=k$ between profile $d_{1}^{(i)}$ and $d_{2}^{(j)}$. (b) Probability for two different fingerprints having exactly $k$ correspondences: $\hat{p}_{\mathrm{k}}$, prof $(k)$ vs. normalized histogram $r(k)$ from $C$.

from $s_{1}^{(i)}$ are compared with all descriptors from $s_{2}^{(j)}$. As the descriptors should only match, if $i=j$ and if their position is not altered, we get an estimate for $p_{\mathrm{fp}}$, desc by counting false positive correspondences $n_{\mathrm{fp}}$ versus true negative correspondences $n_{\mathrm{tn}}$. Regarding $p_{\text {cstl }}$, which is the probability for a random RANSAC inlier, we compare the inlier area $s_{\mathrm{i}}$ with the area of possible feature locations $s_{\mathrm{p}}$. As nearly all mismatches from the descriptor matching occur at small frequency differences, we chose to compare the areas in the $1 \mathrm{D}$ space domain and not in the $2 \mathrm{D}$ space-frequency domain:

$$
\hat{p}_{\mathrm{fp}, \mathrm{desc}}=\frac{n_{\mathrm{fp}}}{n_{\mathrm{tn}}+n_{\mathrm{fp}}}=1.54 \%, \quad \hat{p}_{\mathrm{cstl}}=\frac{s_{\mathrm{i}}}{s_{\mathrm{p}}}=\frac{2 r}{s_{\mathrm{p}}}=0.48 \% .
$$

In Fig. 3a, the confusion matrix $C(i, j)$ with the number of correspondences $k$ between $d_{1}^{(i)}$ and $d_{2}^{(j)}$ is displayed. For enhanced visibility, $C$ is scaled between $k=0$ and $k=10$. Please note that true positives on the diagonal have a higher expected match count of $\mathrm{E}[C(i, i)]=26$. For $i \neq j$, only up to $k=6$ false correspondences occurred with $\mathrm{E}[C(i, j)]=1.9$. The normalized histogram $r(k)$ of $C(i, j)=k$ is displayed in Fig. $3 \mathrm{~b}$. It is visible that our estimate $\hat{p}_{\mathrm{k}, \text { prof }}(k)$ follows $r(k)$. To compare the strength of our approach to natural fingerprints, we extrapolate using $\hat{p}_{\mathrm{k}, \text { prof }}(k)$ up to $k=19$, which is a reasonable value as classification border as the expected true positive match count is significantly higher. We receive a profile false detection probability of $\hat{p}_{\mathrm{fp}, \text { prof }}(k=19) \approx$ $10^{-20}$. Thus, we have shown that such grinding imprints are as strong as human fingerprints for identification.

\subsection{Corrosion}

In this experiment, we analyze the influence of corrosion to the redetection of an imprint. Salt spray corrosion tests (DIN EN ISO 9227) were used to artificially age the component: $1.5 \mathrm{ml} / \mathrm{h}$ of a $5 \% \mathrm{NaCl}$ salt solution are sprayed onto the surface for a certain duration $\tau$ of aging. Afterward, the surface is acid cleaned. From the surface views in Fig. 4, it can be seen, that two different effects occur: 


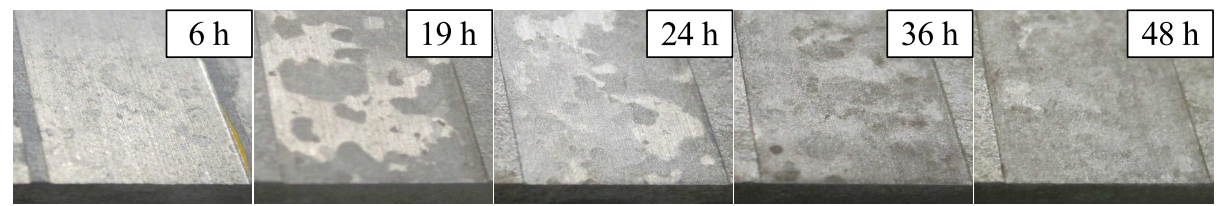

Fig. 4: Corrosion on the grinding imprint after aging for a time span $\tau$.

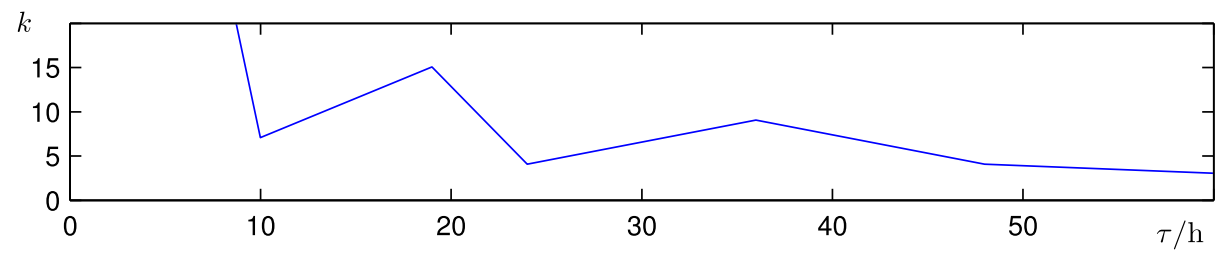

Fig. 5: Number of correspondences $k$ after aging for time span $\tau$.

continuous perturbation of the surface, and the complete destruction of local spots which is best visible for $\tau=19 \mathrm{~h}$. We compare the number of correspondences $k$ between original and corroded imprint over $\tau$ in Fig. 5 .

It can be seen that the outcomes are lowering unsteadily, which is a result of local destruction. However $k$ is still significantly higher than for different imprints as our representation allows partial matches in space and frequency. This can be observed in Fig. 2, in which the correspondences between original and a corrosion of $\tau=19 \mathrm{~h}$ are displayed.

\subsection{Optical Fingerprint Comparison}

In this experiment, we will demonstrate that the here-presented approach is able to identify imprints obtained from the plain image intensities. As input, we use diffusely-illuminated images from the same imprint which were taken with a customary camera at a resolution of $1000 \times 1000 \mathrm{pel}^{2}$. In contrast to Experiment 4.1, we focus on a high detection rate here for an optical on-line comparison, as a positive detection could be verified by a depth map if a low false detection rate is needed. Thus, we set the correspondence threshold to $k=10$, which is reasonable as the number of samples is only one third compared to depth profiles. Apart from this, only $d_{t}$ from (5) was adopted to 0.8 .

In Fig. 6a, images from the same profile with varying brightness, camera pose (up to $10 \%$ shift) and scaling (up to $30 \%$ zoom) were taken. Similar to Experiment 4.1, we match every profile $s_{1}^{(i)}$ obtained from the upper image half to every profile $s_{2}^{(j)}$ from the lower half. In Fig. 6b, the number of correspondences between $i$ and $j$ is displayed. In total, we receive a detection rate of $44 \%$, which is very good for this challenging task. If we fix the pose and only vary the illumination, we even get $77 \%$ detection rate. 


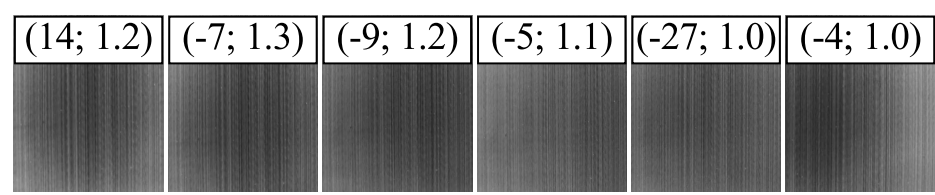

(a)

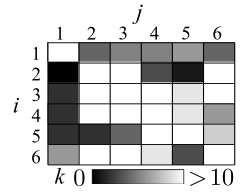

(b)

Fig. 6: (a) Intensity images $d^{(i)}$ with varying camera pose and estimates of $\left(\Delta_{x} / \mathrm{pel} ; \Delta_{f}\right)$ for profile $s_{1}^{(i)}$ with respect to profile $s_{2}^{(6)}$ with smallest zoom. (b) Confusion matrix with number of matches between $s_{1}^{(i)}$ and $s_{2}^{(j)}$.

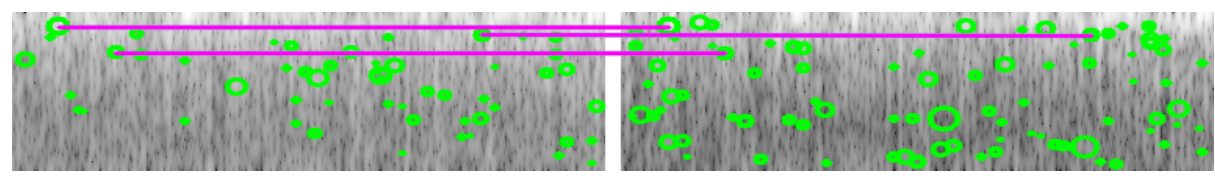

Fig. 7: Depth profile spectrograms of the same surface. Unlike the data of [15], there is no coarse structure which could be used for SIFT matches (Cf. Fig. 2).

\subsection{Comparison to SIFT matching}

We now compare our results to the approach of [15], in which customary SIFT features are used on an audio signal STFT spectrogram. We use the provided parameters and input the depth profiles from Experiment 4.1 instead of audio data. As the signal length is much shorter (3200 samples compared to 441000 for a $10 \mathrm{~s}$ block) and as the profiles are more transient than audio signals, we chose a window length of 32 samples to obtain the spectrogram. Feature extraction and comparison was carried out as described in [15].

In Fig. 7, spectrograms from two measurements $s_{1}^{(i)}$ and $s_{2}^{(i)}$ of the same depth profile are displayed. It can be seen that there are only 3 correspondences detected, which is one of the best results of this method. In total, if profile $s_{1}^{(i)}$ is matched with itself, an average of 84 correspondences were found, if it is matched with the second measurement $s_{2}^{(i)}$ at $1 \mathrm{~mm}$ distance, this value drops to 0.36 (for different profiles $s_{1}^{(i)}$ and $s_{2}^{(j)}$, it is 0.03$)$. This shows that the spectrogram is not suitable to robustly and uniquely describe transient profiles.

\section{Summary and Conclusion}

In this paper we have shown an approach to extract and compare robust and precise characterizations of grinding imprints. It is based on features obtained from the continuous wavelet transformation. This allows a shift and scale invariant characterization of the profile. For the comparison of two fingerprints, the number of corresponding features with consistent geometric constellation is used and compared with a threshold $k$. In experiments we have shown that the 
approach is robust to perturbations like corrosion. We have shown that a similar approach which uses an STFT spectrogram in combination with SIFT is not suited for this data. We further have demonstrated that our approach works on intensity images as well as on depth maps, even under variation of illumination and pose. To estimate the strength of the proposed approach, a model on the false positive detection probability $\hat{p}_{\mathrm{fp}}$, prof $(k)$ was derived and its parameters trained. This model indicates that a reasonable amount of $k \geq 19$ matches leads to as few false positive detections as if human fingerprints were compared.

Acknowledgments: This work was funded by the DFG within the Collaborative Research Centre 653. We thank the Institute of Materials Science of the Leibniz Universität Hannover for their support of artificially aging components. Further we thank the author of the RANSAC implementation [16] that we used.

\section{References}

1. Cano, P., Batlle, E., Kalker, T., Haitsma, J.: A review of audio fingerprinting. The Journal of VLSI Signal Processing 41, 271-284 (2005)

2. Fischler, M.A., Bolles, R.C.: Random sample consensus: a paradigm for model fitting with applications to image analysis and automated cartography. CACM 24, 381-395 (June 1981)

3. Grinsted, A., Moore, J.C., Jevrejeva, S.: Application of the cross wavelet transform and wavelet coherence to geophysical time series. Nonlinear Processes in Geophysics 11(5/6), 561-566 (Nov 2004)

4. Grossmann, P.: Depth from focus. Pattern Recognition Letters 5(1), 63 - 69 (1987)

5. Kadir, T., Zisserman, A., Brady, M.: An affine invariant salient region detector. In: Proc. ECCV. pp. 228-241 (2004)

6. Lowe, D.G.: Distinctive image features from scale-invariant keypoints. International Journal of Computer Vision 60, 91-110 (2004)

7. Mallat, S.: a wavelet tour of signal processing - The Sparse Way. Elsevier (2009)

8. Matas, J., Chum, O., Urban, M., Pajdla, T.: Robust wide-baseline stereo from maximally stable extremal regions. Image and Vision Computing (2004)

9. Mikolajcyk, K., Schmid, C.: An affine invariant interest point detector. In: Proc. ICCV (2002)

10. Murphy, B., Morrison, R.D. (eds.): Introduction to environmental forensics. Academic Press, 2nd edn. (2007)

11. Pankanti, S., Prabhakar, S., Jain, A.K.: On the individuality of fingerprints. Transactions on Pattern Analysis and Machine Intelligence 24, 1010-1025 (2001)

12. Rua, A.: Measuring comovement in the time-frequency space. Journal of Macroeconomics 32(2), $685-691$ (2010)

13. Sporring, J., Weickert, J.: Information measures in scale-spaces. Transactions on Information Theory 45(3), 1051-1058 (Apr 1999)

14. Tuyls, P., Guajardo, J., Batina, L., Kerins, T.: Anti-counterfeiting. In: Tuyls, P., Skoric, B., Kevenaar, T. (eds.) Security with Noisy Data, pp. 293-312. Springer London (2007)

15. Zhu, B., Li, W., Wang, Z., Xue, X.: A novel audio fingerprinting method robust to time scale modification and pitch shifting. In: Proc. ACM International Conference on Multimedia. pp. 987-990 (Oct 2010)

16. Zuliani, M.: Ransac toolbox for matlab (Nov 2008), http://www . mathworks.com/ matlabcentral/fileexchange/18555 